\title{
Physical conditions of the acceleration region of a solar flare with an unusually narrow gyrosynchrotron spectrum
}

\section{Guillermo G. Giménez de Castro, Joaquim E. Rezende Costa and Adriana Válio Roque da Silva}

\author{
Centro de Rádio Astronomia e Astrofísica Mackenzie \\ R. da Consolação 896, 01302-907, São Paulo, Brazil \\ email: guigue@craam.mackenzie.br
}

During the rising phase of the August 30, 2002 X1.5 flare a short pulse with a total duration of 8 seconds was observed. Its background-subtracted radio spectrum ranges only from 5 to $12 \mathrm{GHz}$ with a maximum flux density of approximately 900 s.f.u. at $7 \mathrm{GHz}$ and a steep optically thin spectral index $\alpha \simeq 8$. Maximum degree of polarization at $7 \mathrm{GHz}$ is around $5 \%$. The hard X-ray pulse emission above the background in the range of 30$150 \mathrm{keV}$ observed by RHESSI is coincident in time with the microwave observation. Hard $\mathrm{X}$-ray images reveal very compact $\left(\sim 10^{\prime \prime}\right)$ footpoint sources. Below $30 \mathrm{keV}$, a thermal source is observed.

In order to explain the microwave spectrum we analyzed different alternatives. A thermal source would need extremely high temperature to produce the photons of $150 \mathrm{keV}$ observed with RHESSI. Harmonic or maser emission generate spectra which are narrower than ours, and are strongly polarized. We used instead a distribution of accelerated electrons represented by a double power law, with $\delta_{E}<250 \mathrm{keV}=5.3$ and $\delta_{E} \geqslant 250 \mathrm{keV}=13$, a $35 \mathrm{keV}$ low energy cutoff, and computed the gyrosynchrotron and thick target bremsstrahlung fluxes of a homogeneous source. A thermal source with $E M=10^{48} \mathrm{~cm}^{-3}$ and $T=3.210^{7} \mathrm{~K}$ was added to fit the X-ray spectrum below $35 \mathrm{keV}$.

From our fitting we determine a rate of $\dot{N}=7.510^{34} \mathrm{~s}^{-1}$ accelerated electrons. The power deposition of the nonthermal electrons is $5.510^{28} \leqslant Q \leqslant 5.510^{29} \mathrm{ergs} \mathrm{s}^{-1}$, depending on the plasma density assumed: $10^{10} \leqslant n \leqslant 10^{11} \mathrm{~cm}^{-3}$. The ratio $\dot{N} / Q$ can be related to the accelerating DC electric field. Using the relation established by Holman et al. (1989), we may deduce that $17 \leqslant E_{d} / E \leqslant 24$ here with $E$ the electric field, and $E_{d}$ the Dreicer field. The critical energy above which the electrons runaway can also be determined, yielding $24 \leqslant W_{c} \leqslant 33 \mathrm{keV}$. Finally, the total number of current sheets is $7.510^{4} \leqslant s \leqslant 10^{5}$.

This uncommon event, short in time and intense in flux, is explained by means of a beam of accelerated electrons with energies mainly between 35 and $250 \mathrm{keV}$, because the electron index above the break energy is so high that it acts as a high-energy cutoff. Assuming that a DC field accelerated the electrons, we concluded that the electric field should be sub-Dreicer. The critical energy obtained, around $30 \mathrm{keV}$, is compatible with the $35 \mathrm{keV}$ low-energy cutoff of our model. The large number of current sheets needed to accelerate the electrons could be overestimated if the current sheet density is higher or if the resistivity is non classical.

\section{Reference}

Holman, G. D., Kundu, M. R., \& Kane, S. R. 1989, ApJ, 345, 1050 